\title{
New non-stationary gradient model of heat-mass-electric charge transfer in thin porous media
}

\author{
V. B. Rogankov, M. V. Shvets, O. V. Rogankov \\ Odessa National Academy of food Technologies, 112 Kanatnaya str., Odessa, 65082, Ukraine
}

\begin{abstract}
The well-known complicated system of non-equilibrium balance equations for a continuous fluid $(f)$ medium needs the new non-Gibbsian model of f-phase to be applicable for description of the heterogeneous porous media (PMs). It should be supplemented by the respective coupled thermal and caloric equations of state (EOS) developed specially for PMs to become adequate and solvable for the irreversible transport f-processes. The set of standard assumptions adopted by the linear (or quasi-linear) non-equilibrium thermodynamics are based on the empirical gradient-caused correlations between flows and forces. It leads, in particular, to the oversimplified stationary solutions for PMs. The most questionable but typical modeling suppositions of the stationary gradient (SG) theory are: 1) the assumption of incompressibility accepted, as a rule, for f-flows; 2) the ignorance of distinctions between the hydrophilic and hydrophobic influence of a porous matrix on the properties; 3) the omission of effects arising due to the concomitant phase intra-porous transitions between the neighboring f-fragments with the sharp differences in densities; 4) the use of exclusively Gibbsian (i.e. homogeneous and everywhere differentiable) description of any f-phase in PM; 5) the very restrictive reduction of the mechanical velocity field to its specific potential form in the balance equation of $f$-motion as well as of the heat velocity field in the balance equation of internal energy; 6) the neglect of the new specific peculiarities arising due to the study of any non-equilibrium PM in the meso- and nano-scales of a finite-size macroscopic $(N, V)$-system of discrete particles. This work is an attempt to develop the alternative non-stationary gradient (NSG) model of real irreversible processes in PM. Another aim is to apply it without the above restrictions 1)6) to the description of $f$-flows through the obviously non-Gibbsian thin porous medium (TPM). We will suppose that it is composed by two inter-penetrable fractal sf-structures off-phase (formed by the "mixture" of g-and l-phases termed, in total, interphase) and solid ( $s$ ) porous matrix termed below s-phase. The permanent influence of humidity and the respective increase of the moisture content in TPM including the unavoidable phenomenon of capillary condensation are the main factors to occur the non-stationary transport f-flows through its texture.
\end{abstract}

Key words: thin porous media; non-stationary gradient model; transport process of fractal fluid phases; non-gibbsian heterogeneous structures.

\section{Нова нестаціонарна градієнтна модель переносу тепла, маси і електричного заряду в тонких пористих середовищах}

\author{
В. Б. Роганков, М. В. Швець, О. В. Роганков
}

Одеська національна академія харчових технологій, вул. Канатна, 112, Одеса, 65082, Україна

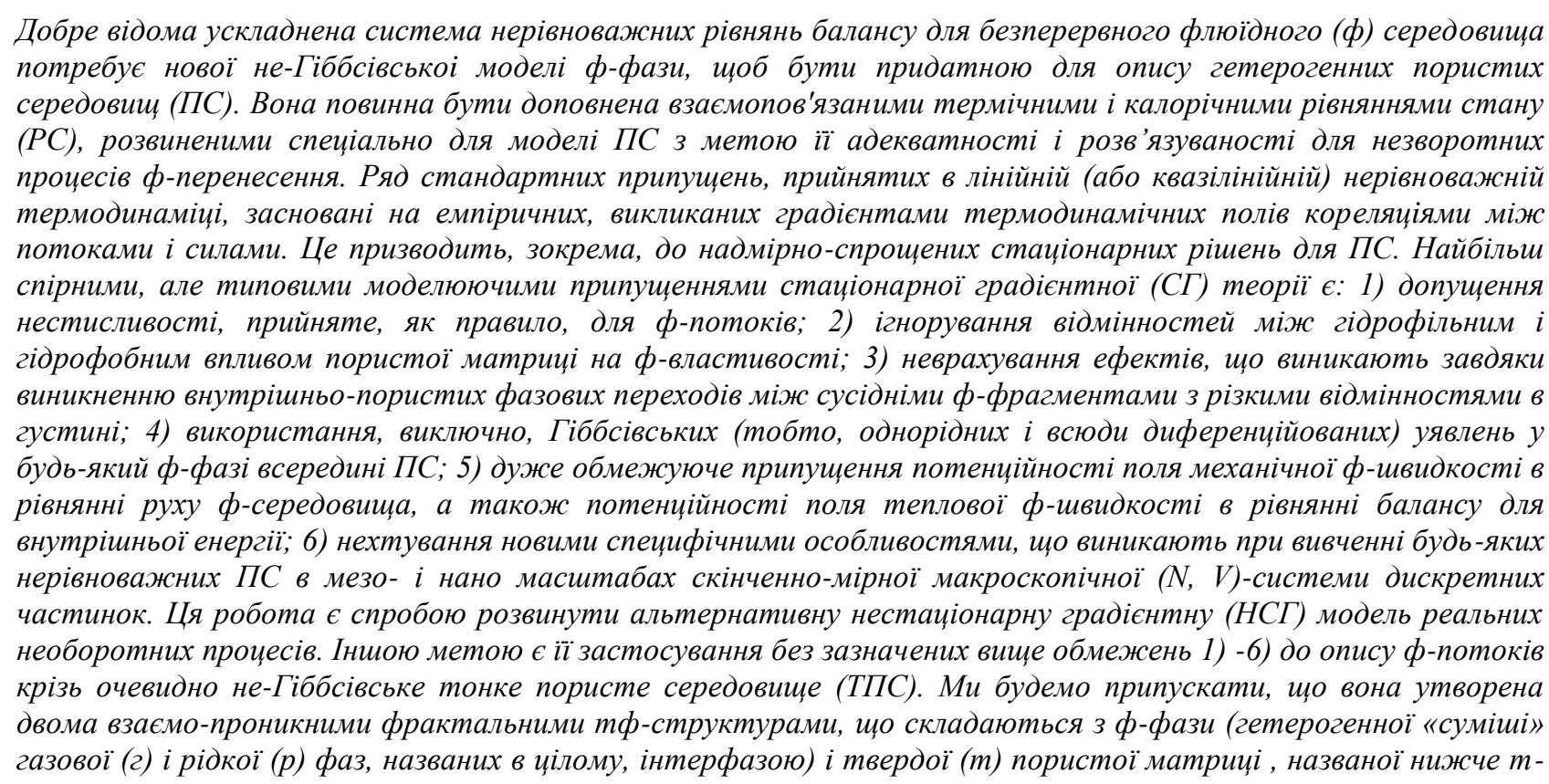


фазою. Постійний вплив вологості і відповідне збільшення вмісту вологи в ТПС, включаючи неминуче явище капілярної конденсачії, слід вважати основними факторами, щэо стимулюють нестаціонарність ф-потоків переносу через її структуру.

Ключові слова: тонкі пористі середовища; нестаціонарна градієнтна модель; процеси переносу в фрактальних флюїдних фазах; не-Гіббсівські гетерогенні структури.

(C) The Author(s) 2017. This article is an open access publication

This work is licensed under the Creative Commons Attribution 4.0 International License (CC BY)

http://creativecommons.org/licenses/by/4.0/

\section{Introduction}

The functioning of a variety of TPMs (thin porous media which share the wide range of thickness $\delta$ from the textile fabrics and the polymer protecting plates up to the walls of buildings) needs the clear recognition of the relevant transport processes. It is hard task even to enumerate the respective experimental and theoretical works considering the one-dimensional heat-and-mass transfer through the usual PM at the given stationary gradients of pressure $\nabla P \equiv(\partial P / \partial x)_{t} \approx \Delta P / L$ and temperature $\nabla T \equiv \equiv(\partial T / \partial x)_{t} \approx \Delta T / L$. Thus, one admits that a sample of PM in the chosen $x$-direction of its fixed thickness $L$ (it is here compatible with two other linear sizes) has the maintained isothermal-isobaric (hence the locally-equilibrium) LE-conditions on its internal $\left(T_{1}, P_{1}\right)$ and external $\left(T_{2}, P_{2}\right)$ sides-planes. Second Law determines, of course, the direction of a resulting forced heat-mass flow in dependence on the given relationship between $T_{1}$ and $T_{2}$ as well as between $P_{1}$ and $P_{2}$. The socalled "dry" air porosity $\varepsilon=V_{d a} / V_{M}<1$ and the certain implied spatial structure of $s$-phase (porous matrix $M$ ) are the determinative factors for any PM at the traditional study of transport processes. The unavoidable moisture content of $f$-phase $\omega=m_{f} / m_{d a}$ is ignorable, as a rule, in such onephase investigations.

The most serious and unsolved until now problem is the necessity to extend such oversimplified stationarygradient model (SG-) on the description of nonstationary processes by the realistic gradient models (NSG). It arises if the initial and boundary conditions become $t$-dependent. Another manifestation of complexity in this problem is an occurrence of a local first-order phase transition (condensation and/or vaporization) in the pores-capillaries induced by their hydrophobic ( $h b-)$ or hydrophilic ( $h l$-) internal surfaces and by the possible change of the applied to PM external $(P, T)$-conditions. One may add to these factors the changeable influence of a relative humidity $\phi$, $\%$ which provides the inflowing moisture air content $\omega(t)$ in any PM. Therefore, itself notion of "dry" porosity $\varepsilon$ loses perceptibly its meaning with a gradual increase of $\omega$ inside of texture. Simultaneously, the respective swelled shape of PM and its mechanic deformation become the essential factors at the description of transport processes.

It was recently shown [1] that all above-mentioned characteristics of a realistic PM can manifest their cumulative effect by the rather simple experimental observation, at least, for the particular case of TPMs (see below). The specific feature of latters is the very small actual magnitude of thickness $(\delta<<L)$ which becomes much less than two other linear TPM-sizes. From a formal viewpoint, all described trends of a supposed NSG-model should be pronounced in such actually one-dimensional transport. Indeed, the thermodynamic forces-gradients become augmented: $\nabla P \approx \Delta P / \delta>>\Delta P / L$ and $\nabla T \approx \Delta T / \delta>\Delta T / L$ in comparison with a usual PM. Moreover, it is naturally to admit the failure for TPM of the non-equilibrium linear SG-model of thermodynamics [2,3]. Formally, the gradients of fields $(\nabla P ; \nabla T ; \nabla \mu-$ of chemical potential, $\nabla \varphi-$ of electrostatic potential) through TPM become great while the respective vector convection of mass $(\rho \vec{u})$ and/or diffusion flow (of momentum $\vec{j}_{m \vec{u}}$, heat $\vec{j}_{Q}$, mass $\vec{j}_{m}$ and free charge $\vec{j}_{q}$ ) cannot be too great to provide its expected proportionality to the above gradients. Oppositely, linear non-equilibrium SG-model postulates that gradients and flows should be small and linearly-dependent. The well-established only in the framework of a bilinear entropy production [2,3] crosseffects of thermo- and electro-diffusion as well as the thermoelectric phenomena must be the non-linearly interdependent in a TPM and the determinative factors for a common transport flow. In other words, the problem of NSG-model becomes so complex in the case of TPM that its any simplified (and, even, rather approximate) solution seems to be very useful for applications.

Additionally, the appearance in the recent years of a "smart-texture's" (ST-) concept applied initially just to the textile fabrics makes the theoretical investigation of TPMs especially actual. The construction for them of an adequate NSG-model confirmed by the relatively scarce and restricted experimental data can lead to the novel insight into ST-problem. The main aim of such investigations is the search for the appropriate controlling parameters and factors.

\section{Reference ideal-liquid and perturbation ideal- liquid with thermal conductivity regimes proposed for NSG-model of heterophase phenomena in any $P M$.}

We refer now the readers of present work to our previous results reported not only in [1] but also in [4-6] where the foundation of fluctuational thermodynamics model (FT- model) has been in detail represented. The main 
idea of latter was the extension of macroscopic nonequilibrium thermodynamics $[2,3]$ on the spatial mesoscopic, nano- and, even, microscopic (i.e. compatible with the effective sizes of atoms, ions and molecules) scales. The implied methodology of such extrapolation maintains the hypothesis of LE-states but formulated, exclusively, in terms of the independent fields $(T, \mu, \varphi)$ and their unique thermodynamic potential $P(T, \mu, \varphi)$. The approach involves their conjugated, strongly fluctuated in PM densities $\rho e\left(\rho s, \rho, \rho e_{q}\right)$ determined per unite of volume by the fundamental LE-condition. It is formulated in FT-model [4-6] by means of the standard [2,3] substantial derivatives $(D / D t=\partial / \partial t+\vec{u} \cdot \partial / \partial \vec{r})$ for the velocity field $\vec{u}(\vec{r}, t)$ :

$$
\frac{1}{\rho} \frac{D P}{D t}=s \frac{D T}{D t}+\frac{D \mu}{D t}+e_{q} \frac{D \varphi}{D t},\left[\frac{W}{k g}\right],
$$

where $s=S / m$ is the specific (per unit of mass) entropy and $e_{q}=q / m$ is the specific charge ( $e$ denotes below the specific internal energy $E / m$ ).

To avoid the misunderstandings, let us note that the used also denotation $D_{m}$ is related, mainly, to the isothermal-isobaric mass diffusion coefficient $\left[\mathrm{m}^{2} / \mathrm{s}\right]$ in the Fick's law for the density gradient $\nabla \rho\left[\mathrm{kg} / \mathrm{m}^{4}\right]$. This "force" as well as the similar, widely usable moisture content gradient $\nabla \omega[1 / m]$ (see Eqs. $(2,3)$ ) exist in the interfacial layers finite thickness of the first-order vaporliquid $(v, l)$ VLE-equilibrium phase transition. Hence, both ones correspond to the equality of chemical potential $\mu_{v}(P, T)=\mu_{l}(P, T)$ and themselves cannot obligatory lead to the thermodynamic irreversibility of such heterophase self-diffusion. The real cause for latter may be only gradient $\nabla \mu\left[\mathrm{m} / \mathrm{s}^{2}\right]$. Its dimensionality prompts to many authors the questionable idea at the formulation of LE-hypothesis in terms of the co-called modified fluidpressure: $P=P_{f}+\rho_{f} g \cdot z(f=v$ or $l)$. It is composed by the omnidirectional scalar $\left(P_{f}\right)$ and the strictly vector $\left(\rho_{f} \vec{g} z\right)$ components (see, for comparison, Eq.(4)). Such combination of external gravitation field and molecularbased internal field seems to be adequate at the description of a convection flow by the known Bernoulli's integral (see below). However, the similar estimate of the diffusive, by nature, velocity field described by the famous D'Arcy's law for an irreversible barodiffusion through PM seems to be oversimplified. One should take into account the quite different influence of gravity on the air flow (where it is negligible) and on the liquid flow (where it is essential).

The accepted form of LE-hypothesis in Eq.(1) emphasizes the determinative scaling meaning of the inhomogeneous mass density $\rho(x, t)$. It leads, in particular, to a possibility of the automodel solution in terms of the diffusion scaled variable $\sqrt{D_{m}} \sim \sqrt{x^{2} / t}$ for the balance equation of total mass $m$. Unfortunately, both alternative and widespread differential NSG-forms [7-15] of this equation for PMs based on the strongly fluctuating field of a one-dimensional moisture content $\omega(x, t)$ seems to be hardly adequate for TPMs:

$$
\begin{gathered}
\frac{\partial \omega}{\partial t}=\vec{\nabla} \cdot\left(D_{\omega} \nabla \omega+D_{T} \nabla T+D_{\varphi} \nabla \varphi+D_{g} \nabla z\right), \\
\frac{\partial \omega}{\partial t}=\vec{\nabla} \cdot(D\{\omega(x, t)\} \nabla \omega),\left[\frac{1}{s}\right],[10-15],
\end{gathered}
$$

where the difference between the gradient applied to the modeling vector velocity field $\vec{\nabla} \cdot \vec{u}(x, t)$ (i.e. its divergence) and the gradient applied to the scalar potential fields $\nabla \omega, \nabla T, \nabla \varphi, \nabla z$ is emphasized by arrow placed over the former. The similar distinction has been also used by FT-model for the divergence of the vector heat flow $\vec{\nabla} \cdot \vec{j}_{Q}$ included in both coupled balance equations for $E$ (internal energy) and $S$ (entropy).

In opposite to the vast majority of known solutions performed for Eqs. $(2,3)$, the stringent thermodynamic hierarchy of the determinative sequence has been introduced by FT-model for a chosen here set of physical fields $\{P \rightarrow T \rightarrow \mu, \varphi \rightarrow \vec{g}, \vec{E}, \vec{u} \times \vec{B}\}$. Tensities of the fundamental gravitation $\vec{g}\left[\mathrm{~m} / \mathrm{s}^{2}\right]$ and electromagnetic $\left\{\vec{E}=-\nabla \varphi[\mathrm{J} / \mathrm{Cl} \cdot \mathrm{m}], \vec{B}\left[\mathrm{~J} \cdot \mathrm{s} / \mathrm{Cl} \cdot \mathrm{m}^{2}\right]\right\}$ fields determine the relatively small external force-field influence per unit of volume $f^{\text {ext }} / V$ on the moving fluid:

$$
\rho \frac{D \vec{u}}{D t}=-\nabla P+\frac{1}{V}\left(m \vec{g}+q \vec{E}+q \vec{u}_{q} \times \vec{B}\right)^{e x t}\left[\frac{N}{m^{3}}\right]
$$

The further FT-transformation of two main Eulerian regimes for the above hierarchy has the following meaning and value illustrated schematically by the reported below sequence of steps. The first reference regime is introduced as an alternative to the widely usable in the phenomenology of heat and mass transfer EOS-models of ideal gas and of its mixtures. An appearance of time $t$ [s] in Eq.(1) is the realistic feature at the study of actual non-equilibrium processes in the finite volumes $V\left[\mathrm{~m}^{3}\right]$ of any locally heterogeneous (i.e. non-Gibbsian) N-systems [4-6].

\section{IL-regime of NSG-model for a reference compressible ideal liquid without the thermal conductivity and the viscous damping (induced by the D'Arcy's law)}

\section{Assumptions:}

1a) the common density of external forces in Eq.(4) tends to zero $f^{\text {ext }} / V \rightarrow 0$.

1b) the adopted approximation of isotropicity in Eq.(4) for the tensor of deformation: $P_{i j}=-\delta_{i j} P\left(\delta_{i j}=0\right.$ at $i \neq j$ ) provides the LE-interpretation of $P$;

1c) the implied identification of the resulting Eulerian Eq.(4) with the vector equation of motion in the Newton's mechanics $(D / D t \Leftrightarrow d / d t)$ for the conservative field of a potential pressure-field $P(x)$ per unit of volume. 


\section{Consequences and results}

1A) the introduction by FT-model of the dimensionless "thermodynamic time" for PM (and for any other inhomogeneous media) is postulated by the equality including the inverse volume:

$$
\begin{aligned}
& t_{V}=V_{0} / V \\
& d t_{V} / t_{V}=-d V / V=-(\vec{\nabla} \cdot \vec{u}) d t
\end{aligned}
$$

Its aim is the adequate account for the elasticity of medium by the isothermal or isoentropic bulk modulus $B_{T, s}$ (or by the respective compressibility:

$$
\begin{aligned}
& \left.\chi_{T, s}=(1 / \rho)(\partial \rho / \partial P)_{T, s} \equiv 1 / B_{T, s}\right): \\
& \frac{\Delta P}{L^{2} \vec{\nabla} \cdot \vec{u}}=-B_{T, s} \frac{\Delta t}{L^{2}} \equiv-\frac{\Delta t}{\chi_{T, s} L^{2}},\left[\frac{P a \cdot s}{m^{2}}\right],
\end{aligned}
$$

where the usual physical time $\Delta t=t-t_{0}$ and the pressure drop through PM $\Delta P=P-P_{0}$ are the finite $\Delta$-differences which have been used instead of differentials $d$ for the simplicity and for the further correlation with Eq.(1);

1B) Since the relative "thermodynamic time" is now presented as the ratio $(\Delta \rho / \rho)_{T, S}$ in both characteristics of elasticity $B_{T, s}$ and $\chi_{T, s}$, further elimination of $(\vec{\nabla} \cdot \vec{u})$ divergence from the system composed by the Eulerian's equation of motion and by the continuity equation provides straightforwardly the integrated $\rho_{T, s}(P)$-dependences:

$$
\begin{aligned}
& \rho=\rho_{0} \exp \left(\chi_{T, s} \Delta P_{T, s}\right) \quad(a) \\
& \Delta \rho=\rho_{0}\left[\exp \left(\chi_{T, s} \Delta P_{T, s}\right)-1\right] \quad(b)
\end{aligned}
$$

1C) An absence of the viscous damping and the thermal conductivity postulated for the ideal liquid corresponds to the following isoentropic integral of motion in the Eulerian description $Z_{s}^{I L}(t) \approx 0$ (which defines here the negligible entropy production $[2,3])$ :

$$
\begin{aligned}
& Z_{s}^{I L}(t) \equiv \rho \frac{D s}{D t} \equiv \\
& \equiv \rho\left(\frac{\partial s}{\partial t}+\vec{u} \cdot \nabla s\right)=0\left[\frac{J}{m^{3} K \cdot s}\right]
\end{aligned}
$$

1D) Its usage in a combination with the LE-hypothesis of Eq.(1) leads immediately to the generalized Bernoulli's integral:

$$
\begin{aligned}
& \rho\left(\frac{u^{2}}{2}+\mu+e_{q} \varphi+s T\right)= \\
& =\rho\left(\frac{u^{2}}{2}+h\right)=\text { const },\left[\frac{J}{m^{3}}\right]
\end{aligned}
$$

which is (again formally) identified by FT-model with the total "mechanical" energy (i.e. with the "hamiltonian" of mechanics) for a conservative "potential" enthalpy field $\rho h(P, s)\left[\mathrm{J} / \mathrm{m}^{3}\right]$ per unit of volume:

$$
\rho h(P, s)=\rho e(v, s)+P, \quad\left[\frac{J}{m^{3}}\right] .
$$

1E) The IL-regime admits that any arbitrary path of the possible perturbation non-equilibrium processes (see below regime ILT) can be adequately expressed in terms of two experimentally controllable caloric and thermal EOSs:

$$
\begin{gathered}
\rho h(P, T)=\rho e(v, T)+P(v, T), \\
\Delta h=C_{P}(P, T) \Delta_{P} T \quad(a) \\
\Delta e=C_{v}(v, T) \Delta_{v} T \quad(b)
\end{gathered}
$$

where $C_{P}[\mathrm{~J} / \mathrm{kg} \mathrm{K}]$ and $C_{v}[\mathrm{~J} / \mathrm{kg} \mathrm{K}]$ are the standard heat capacities. Hence, to construct the thermodynamic description of medium one should also know both $f$ - and $s$ types of EOS for any non-Gibbsian (i.e. fractal by its nature) complex phase. It is composed by the fractal solid (s) matrix of PM and by the fractal fluid (liquid and/or gas) flows moving inside of it. FT-model imitates both ones by the molecular-based concept of an excluded volume introduced long ago by van der Waals.

Namely the alternative to entropy from Eq.(10) value of temperature in the system of Eqs. $(11,12)$ stands it on the second place (after the pressure) in the above thermodynamic hierarchy of physical fields at the description of real irreversibility. To corroborate such a special role of $T$ and of its conjugated variable of entropy $s$ for any non-equilibrium process, let us remind that just this pair of isolines was chosen by Carnot to form the wellknown reversible cycle. It was supposedly realized by the extremely (infinitely) quick processes at $s=$ const and by the extremely (infinitely) slow processes at $T=$ const. Strictly speaking, a literal recognition of such extremes should lead to the certain inconsistency between the differential forms of First and Second Laws for the reversible processes (all realistic processes are occurred, of course, during the finite time intervals):

$$
\begin{gathered}
d E=\delta Q-\delta W, \quad\{m, q\}=\text { const },[\mathrm{J}] \\
\int \frac{\delta Q}{T}=0\left[\frac{J}{K}\right] .
\end{gathered}
$$

Another questionable extreme of Carnot's ingenious cycle is, of course, the choice of an ideal gas ( $i g$ ) as one-phase working medium. Its density and pressure $\rho_{i g}\left(T, P_{i g}\right)=P_{i g} M / R T$ should simultaneously tend to zero (to be, in fact, negligible) in accordance with the respective ig-EOS, while the specific ig-internal energy from Eq.(13) $e_{i g}=(3 / 2) R T / M$ depends exclusively on the temperature.

To avoid such oversimplifications, the strategy proposed long ago by FT-model [4] seems to be the most appropriate. Indeed, due to its realization one does not omit on the ad hoc basis the divergences of the vector velocity field $\vec{\nabla} \cdot \vec{u}(x, T)$ in IL-regime and of the directed heat flow $\vec{\nabla} \cdot j_{Q}(x, T)$ in ILT-regime below induced by a gradient of temperature $\nabla T$. Instead of this, it is naturally to 
eliminate their finite (i.e. unknown but realistic) values from the explicit sequence of thermodynamically-consistent transformation steps by the following scheme.

ILT-regime of NSG-model for a compressible ideal liquid with the perturbation contribution of thermal conductivity but without the viscous damping implied by the D'Arcy's law

\section{Assumptions:}

1a) the system of balance equations for entropy and internal energy leads to the irreversible production of entropy $Z_{s}(t)$ which becomes the changeable and $x$, $t$-dependent in comparison with Eq.(8) assumed for IL-regime:

$$
Z_{s}^{I L T}(t)=\rho \frac{D s}{D t}=-\frac{1}{T} \vec{\nabla} \cdot \vec{j}_{Q}(x, t) ;
$$

1b) FT-model proposes to eliminate the itself divergence $\vec{\nabla} \cdot \vec{j}_{Q}$ from the above system of balance equations instead of an attempt to obtain the implied complex solution for the respective parabolic equation of thermal conductivity:

$$
\vec{\nabla} \cdot(\lambda \nabla T)=\left(\rho C_{P, v}\right) \frac{\partial T}{\partial t}-T \cdot Z_{s}^{I L T}(t),
$$

where the subscript $P, v$ for $C_{P, v}$ is recognizable;

1c) the fundamental exact result [4] of such elimination introduces the characteristic relaxation time-scale $\tau[\mathrm{s}]$ for any PM and/or TPM:

$$
Z_{s}^{I L T}=Z_{s}^{I L T}(t=o) \exp [-2 t / \tau],
$$

which is the main parameter of ILT-non-stationary changes; 1d) the necessary estimate of initial $Z_{s}^{I L T}(t=o)$-value can be obtained by solution of the much more simple elliptic (stationary) variant of parabolic Eq.(16) in which the entropy density $\rho \cdot s(x)$ and the relaxation parameter $\tau$ should be preliminarily found:

$$
\vec{\nabla} \cdot(\lambda \nabla T)=-(\rho s T)_{0} / \tau-T_{0} \cdot Z_{s}^{I L T}(t=0)
$$

\section{Consequences and results}

1A) the discussed Poison's-type Eq.(18) for the T-field may be, of course, supplemented by the similar equations for the $\mu$-field and $\varphi$-field following from the LE-condition assumed by Eq.(1):

$$
\begin{aligned}
& \vec{\nabla} \cdot\left(\gamma_{m} \nabla \mu\right)=-\rho_{0} / \tau \quad(a) \\
& \vec{\nabla} \cdot\left(\sigma_{q} \nabla \varphi\right)=-\left(\rho_{0} e_{q}\right)_{0} / \tau \quad(b)^{\prime}
\end{aligned}
$$

where coefficient of the mass conductivity $\gamma_{m}\left[\mathrm{~kg}^{2} / \mathrm{m} \cdot \mathrm{s} \cdot \mathrm{J}\right]$ and the electric conductivity $\sigma_{q}\left[\mathrm{Cl}^{2} / \mathrm{m} \cdot \mathrm{s} \cdot \mathrm{J}\right]$ have been introduced. Their subordinated role in relation to the $T$-field and the use of common $\tau$-value correspond to the abovementioned hierarchy of fields: $P \rightarrow T \rightarrow \mu, \varphi \rightarrow \vec{g}, \vec{E}, \vec{u}_{q} \times \vec{B} ;$

1B) the respective cross-effects leading to the known transport equations of thermal diffusion (Soret effect), thermal-electric diffusion (Peltier effect) and electric diffusion (Nernst effect) may be straightforwardly analyzed for the respective set of quasi-linear $T$-dependent SGcoefficients:

$$
\begin{array}{ll}
j_{m}=-\gamma_{m}(T) \nabla \mu & (a) \\
j_{q}=-\sigma_{q}(T) \nabla \varphi & (b)
\end{array}
$$

supplemented by two measurable linear SG-correlations for thermal conductivity (Fourier's law) and barodiffusion (D'Arcy's law):

$$
\begin{array}{ll}
j_{q}=-\lambda \nabla T & (a) \\
j_{m}=-\tau \nabla P & (b)
\end{array}
$$

1C) the rejection from the combined definition of an electrochemical potential $\left(\mu+e_{q} \varphi\right)$ in Eq.(1) and the remarkable "flexibility" of adopted by FT-model LEcondition which can be represented in terms of the GibbsDuhem's finite differences:

$$
\Delta P=\rho s \Delta T+\rho \Delta \mu+\rho e_{q} \Delta \varphi
$$

Leads immediately to the following SG-description of thermal-electric diffusion for any $f$-and/or s-phase:

$$
\left[\frac{\lambda}{\sigma_{q}(T)}\right]_{f, s}=\left(\frac{s}{e_{q}}\right)_{0}^{2} \cdot T .
$$

It seems that the well-known Videmann-Frantz's law obtained for the proposed by Zommerfeld explanation of the Lorentz's coefficient $L_{s}$ :

$$
\left[\frac{\lambda}{\sigma_{q}}\right]_{s}=L_{s} T=\frac{\pi^{2}}{3}\left(\frac{k_{B}}{e_{-}}\right)^{2} \cdot T .
$$

can be considered as the limiting discrete form of the more general FT-Eq.(23) derived here for the thermal-electric phenomena;

1D) the similar FT-correlation for the electric diffusion has the especially simple form expressed in terms of the specific charge $e_{q}=q / m$ :

$$
\left[\frac{\sigma_{q}(T)}{\gamma_{m}(T)}\right]_{f, s}=\left(e_{q}\right)_{0}^{2} .
$$

It is interesting and informative to compare this result with the well-known Nernst-Einstein's law expressed in terms of the particle concentration $n=N / V$, elementary electric charge $q_{0}$ and the self-diffusion coefficient $D_{m}$ from the Fick's law:

$$
\begin{aligned}
& {\left[\frac{\sigma_{q}}{D_{m}}\right]_{f, s}=\frac{n q_{0}^{2}}{k_{B} T} \quad(a)} \\
& j_{m}=-D_{m} \nabla \rho \quad(b)
\end{aligned} .
$$

Such comparison provides immediately the following explicit $T$-dependence of $\gamma_{m}(T)$-coefficient from Eqs.(20,25):

$$
\gamma_{m}(T)=\rho_{0}^{2} D_{m} /\left(n k_{B} T\right)
$$

as well as the generalized FT-correlation for electric diffusion:

$$
\left[\frac{\sigma_{q}(T)}{D_{m}}\right]_{f, s}=\frac{\left(e_{q} \rho\right)_{0}^{2}}{n k_{B} T}
$$


Its physic sense is obvious: the more is temperature at any density of electric charges, the worse becomes the electric conductivity of a medium;

1E) the combined test of the previous 1C)- and 1D)-results leads to the following FT-correlations for the thermal diffusion:

$$
\begin{aligned}
& {\left[\frac{\lambda}{\gamma_{m}(T)}\right]_{f, s}=s_{0}^{2} T \quad(a)} \\
& {\left[\frac{\lambda}{D_{m}}\right]_{f, s} \frac{(s \rho)_{0}^{2}}{n k_{B}} \quad(b)}
\end{aligned}
$$

For comparison, the formal elimination of $\sigma_{q}$ from the Videmann-Frantz's and Nernst-Einstein's laws leads to the much more restrictive description of the thermodiffusion ratio from Eq.(29b):

$$
\frac{\lambda_{s}}{D_{m f}}=\frac{\pi^{2}}{3} k_{B} n
$$

because it ignores, in fact, the physical reason of thermal conductivity (i.e. transport of heat which is related just to the entropy density $\rho s$ ).

The physical adequacy, simplicity and the experimental testability of the reported FT-correlations are the main advantages used in this work to construct the solvable TPM-model in Sects. 3,4. Let us discuss below, for comparison, the conventional theoretical and simulation approaches to the same or similar PM-problems. The interested reader can find the more detailed description in the cited references [7-9, 22-29]. Our aim here is to emphasize the distinctions between the conventional PMmodels and the proposed NSG-model without the detailed additional comments. We have changed some denotations of the original references to make the comparison more informative.

There are two main concepts in the discussed problem, which can be termed one-medium and two-medium approaches. The former adopts the local thermal equilibrium for the volume-average fields of $f$-velocity $\left\langle u_{f}\right\rangle$ and both main thermodynamic fields $\langle T\rangle,\langle P\rangle$. The abovementioned modified $f$-pressure determines the socalled D'Arcy's $f$-velocity $u_{D}$ namely by the generalized D'Arcy's law:

$$
\begin{aligned}
& P=P_{f}+\rho_{f} g z \quad(a) \\
& u_{D}=\left\langle\vec{u}_{f}\right\rangle=-(K / \eta) \nabla\langle P\rangle
\end{aligned}
$$

where $K\left[\mathrm{~m}^{2}\right]$ is permeability and $\eta[\mathrm{Pa} \cdot \mathrm{s}]$ is viscosity. In accordance with our previous criticism, this approach combined with the further omission of the $f$-velocity divergence $\left(\vec{\nabla} \cdot\left\langle\vec{u}_{f}\right\rangle=0\right)$ leads to the unrealistic model of incompressible $\left(\rho_{0}=\right.$ const $) f$-flow (see, for comparison, FT-Eqs.(5-7)). This conclusion is also related to the "old" conventional interpretations of the D'Arcy's law termed, respectively, the drag theory and the hydraulic radius theory. They were described comprehensively by Iberall long ago [30] for usual PMs:

$$
j_{m}=\frac{\rho_{0} \Delta V}{A \Delta t}=\frac{K}{v} \cdot \frac{\Delta P}{L}\left[\frac{k g}{m^{2} s}\right] .
$$

The D'Arcy's correlation (developed for the flow of liquid water through sands) resembles, of course, the Poiseuille's law for the laminar flow of a continuous liquid through the cross-section area $\pi R^{2}$ proposed to determine viscosity $\eta=\rho_{0} v[\mathrm{~Pa} \cdot s]$ from experiment (see also Eq.(31b)):

$$
j_{m}=\frac{\rho_{0} \Delta V}{\pi R^{2} \Delta t}=\frac{R^{2}}{8 v} \cdot \frac{\Delta P}{L} .
$$

The conjectural replacement of the $\left(R^{2} / 8\right)$-quantity in a medium by the effective quantity termed permeability of PM $K\left[\mathrm{~m}^{2}\right]$ leads, often, to the confusions at its experimental determination and interpretation [1]. The additional, rather crude, from our viewpoint, approximations of the supposed convective velocity $u_{D}$ (termed the D'Arcy's velocity) and the conjectural Reynold's number for PM:

$$
u_{D}=\frac{\Delta V}{A \cdot \varepsilon \cdot \Delta t} \quad \text { (a) } \quad R e^{P M}=\frac{d \cdot u_{D} \cdot \rho_{0}}{\eta}
$$

provide two alternative modeling variants of $K / v$-ratio from Eq.(32):

$$
\begin{gathered}
\rho_{0} u_{D}=\left[\frac{3 d^{2}}{16 v(1-\varepsilon)} \cdot \frac{\left(2-\ln R e^{P M}\right)}{\left(4-\ln R e^{P M}\right)}\right] \cdot \frac{\Delta P}{L}, \\
\rho_{0} u_{D}=\left[\left(\frac{O}{S}\right)^{P M} \frac{r^{2}}{v} \frac{1}{(1-\varepsilon)^{2}}\right] \cdot \frac{\Delta P}{L} .
\end{gathered}
$$

The former expression of the drag theory of permeability modified by Iberall [30] contains "the dimension characteristic of the medium structure, for instance, it is (either) the diameter $(d)$ of the granule (or) the fiber diameter". In accordance with assumptions of Eq.34 (a,b) this is very complicated and implicit for the input parameters $\left(d, \varepsilon, \rho_{0}, \eta\right)$ equation of the stationary modeling D'Arcy's convective flow $\left(\rho_{0} u_{D}\right)$. Such rather formidable drag model is best applicable at high porosities ( $\varepsilon$ belongs to the range of 0.7 to 0.9 ). The latter expression of the hydraulic radius ( $r$ ) theory of permeability developed, mainly, by Kozeny contains the purely adjustable ratio of "an orientation PM-factor $O$ to a shape $P M$-factor $S$ ". It turns out [30] that the hydraulic radius model is applicable exclusively at low porosities in range $\varepsilon$ of 0.1 to 0.3 .

After such interpretation of the mechanical D'Arcy's flow contribution, the

volume-averaged balance energy equation becomes [2-5] a variant of Eq.(16):

$$
\begin{aligned}
& \left\{\sum_{i=s, f}\left(\rho_{0} C_{P}\right)_{i} \varepsilon_{i}\right\} \frac{\partial\langle T\rangle}{\partial t}+ \\
& +\left(\rho_{0} C_{P}\right)_{f}\left\langle u_{f}\right\rangle \cdot \nabla\langle T\rangle-\sum_{i=s, f}\left\langle\dot{s}_{i}\right\rangle= \\
& =\vec{\nabla} \cdot\left[\left(\lambda_{e}+\lambda_{d}\right) \cdot \nabla\langle T\rangle\right]\left[\frac{W}{m^{3}}\right]
\end{aligned}
$$


where two last terms in the left-hand-side provide a combination of the convection contribution (?) for the vector $\nabla T$-field with the "volume-averaged heat source term that can be used to describe the cure kinetics (?) of the resin" [24]. The tensors of the effective thermal conductivity $\lambda_{e}$ and of the thermal dispersion $\lambda_{d}$ introduced on the ad hoc basis in the right-hand-side of Eq.(37) make, to our mind, any its solution to be rather arbitrary.

We have not reported here the even more formidable system of two coupled phase-averaged $f$, $s$-energy equations proposed for the two-medium treatment of PMs-data [2629]. It is hardly to discuss the quite complex differential equations in which the vast majority of input parameters either unknown or determinable with the large uncertainties. The concept of two different $\left\langle T_{f}\right\rangle$ and $\left\langle T_{s}\right\rangle$ temperatures itself seems to be rather questionable if the medium approach is realizable. Nevertheless, we will demonstrate below (Section 3) that the similar discontinuities of the pressure $\Delta P$ and temperature $\Delta T$ but observable within the same f-phase are unavoidable in the realistic $f$-flows through TPM.

This observation returns us to the problem of equilibrium first-order (I) VLE-phase transition arising within any realistic PM due to the appearance of moisture content $\omega$ in its porous texture. We consider, however, the conventional approach [7-9] based on the attempt to model, separately, both $v$ - and $l$-components of the common $f$ velocity field $u_{f}$ as the purely mechanical methodology (proposed long ago by Clebsh):

$$
\begin{aligned}
& \vec{u}_{f}=D_{\omega}^{f} \nabla \omega+D_{T}^{f} \nabla T= \\
& =\left(D_{\omega}^{v}+D_{\omega}^{l}\right) \nabla \omega+\left(D_{T}^{v}+D_{T}^{l}\right) \nabla T .
\end{aligned}
$$

The substitution of such superposition into Eq.(2) leads, simultaneously, to the following modification of the energy balance equation [7-9] in comparison, for example, with the one medium description of Eq.(37):

$$
\begin{aligned}
& (\rho C)^{P M} \cdot \frac{\partial T}{\partial t}- \\
& -\rho_{0} \Delta h_{v}\left[\vec{\nabla} \cdot\left(D_{\omega}^{v} \nabla \omega+D_{T}^{v} \nabla T\right)\right]=, \\
& =\vec{\nabla} \cdot\left(\lambda^{P M} \nabla T\right)
\end{aligned}
$$

where the latent heat $\Delta h_{v}[\mathrm{~J} / \mathrm{kg}]$ (enthalpy of $v \rightarrow l$ change) is related to the capillary condensation and plays, formally, the role of a heat source term. Any solution of the coupled Eqs. $(2,39)$ depends completely on the chosen (supposedly experimental) magnitudes of ill-founded coefficients: $D_{\omega}^{f}, D_{T}^{f}, \ldots$ and $\lambda^{P M}$ related to the "dry" porosity $\varepsilon\left[\mathrm{m}^{3} / \mathrm{m}^{3}\right]$ of PM. In total, the described methodology of I-phase transition in PMs' bears the strong resemblance with the known Clebsh's potential of the convection velocity fields [4]. One may introduce them by Eq.(1) without the above complexities:

$$
\vec{u}_{f}=\left[\nabla \mu+s \nabla T+e_{q} \nabla \varphi\right] \tau,[\mathrm{m} / \mathrm{s}]
$$

\section{Second law and zero-order (0) VLE-transition in non-Gibbsian $f$-phases}

To go beyond the Carnot's cycle artificial extremes, FT-model introduces not only the described reference ILregime with the zero entropy production $Z_{s}^{I L}(t)=0$ of Eq.(8). It uses also the following alternative formulation of Second Law proposed long ago by Joule, Horstmann and others [16]:

$$
\frac{\delta Q}{T}=\frac{d P}{d T} \delta V,
$$

where $\delta V$ is a variation of volume. It emphasizes here the discrete nature of description accepted for $\delta Q$ on the molecular and/or atomic levels. Again let us remind (to avoid the misinterpretation) that the same symbol $\delta$ is used in the present work for the thickness of TPM and to distinct it from the large thickness $L$ of usual PM. Due to such distinction, we have postulated the principal anisotropy of one-dimensional transport flows in which the above "thermodynamic time" of Eq.(5) should be replaced for TPM by the one-dimensional ratios: $t_{\delta}=\delta_{0} / \delta$, $d t_{\delta} / t_{\delta}=-d \delta / \delta=-\left(\vec{\nabla}_{x} \cdot \vec{u}_{x}\right) d t . \quad$ The similar transformation will be used for the linear compressibility $\chi_{\delta} \Delta P=-\Delta \delta / \delta_{0} \quad$ and linear expansivity $\alpha_{\delta} \Delta T=\Delta \delta / \delta_{0}$ of TPMs. From the viewpoint of FTmodel [4-6] both variations in Eq.(41) should be considered as the quantisized finite values (i.e. the quantities composed by the great but finite number of the microscopic portions of energy $k_{B} T[\mathrm{~J}]$ and volume of particles $\left.\left[b \sim d^{3}\right]\left[\mathrm{m}^{3}\right]\right)$.

The presence in the right-hand side of Eq.(41) the only parameters of a ther-mal EOS $(P, V, T)$ supports the above-mentioned concepts of FT-model. The me-chanical theory of heat assumes in this formulation (without any appeals to the unrealistic extremes) that the internal energy content and/or the enthalpy content in Eqs.(10-12) of a body during its state change can be calculated through nothing more than the P,V,T-information. Hence, the heat quantity consumed during any such change is irrespective of what are the path (usually, an isoline) and the thermodynamic cause (usually, a gradient) for this consumption.

Particularly, Joule corroborated the consequence of Second Law by the experimental adiabatic compression of different liquids [16]:

$$
\Delta_{s} T=\left(\frac{T \alpha_{P}}{\rho C_{P}}\right) \Delta_{s} P,
$$

where $\alpha_{P}=-(1 / \rho)(\partial \rho / \partial T)_{P}$ contains, again, the "thermodynamic time" introduced by FT-model. All processes are here realizable during the finite physical time-intervals $\Delta t$. The main conclusion from the Second Law is that the adiabatic compression of a liquid: $\Delta_{s} P>0$ should always lead either to its heating $\Delta_{S} T>0$ if 
$\alpha_{P}>0$ or to its cooling $\Delta_{s} T<0$ if $\alpha_{P}<0$ (as it is in water at temperatures below than: $t=4{ }^{\circ} \mathrm{C}$, for example).

The meaning of the generalized Bernoulli's integral from Eq.(9) becomes now recognizable for any PM. It is the derived in the present work thermodynamic extension of its traditional mechanical value [3] (see our comments to Eq.(1)):

$$
\rho_{0}\left(\frac{u^{2}}{2}+g z\right)+P=\text { const }\left[\frac{J}{m^{3}}\right] .
$$

The latter implies: a) the stationary, non-viscous laminar flow of incompressible ( $\rho_{0}=$ const ) liquid (or, even, gas) in the external gravitation field $\vec{g}$; b) the absence of the internal energy and heat content $h$ (enthalpy) contributions; c) the implied absence of the permeability for the fluid particles in its imaginary walls. One may conclude that the more is a convection contribution in the brackets, the less becomes a molecular momentum flux (pressure) in such rather restrictive and purely mechanical integral of a fluid motion.

The NSG-model rejects in its IL-regime all above assumptions a), b), c) and proposes the much more flexible description of IL-flow through any PM. In this case, the more is a convection non-stationary contribution $u^{2}(t) / 2$, the less becomes a heat content $h(t)$ composed by the interchangeable internal energy $e(t)$ - and pressure $P(t)$-components. However, this interconnection of "kinetic" and "potential" parts of "hamiltonian" in the brackets of Eq.(9) should be additionally controllable by its changeable mass per unit of volume(i.e. by $\rho(t)$ ). Besides, the dynamical nature of such changes in the LE-state should take into account not only the compressibility of IL-flow by Eq.(7) but also the possibility of internal vapor-liquid $(v \rightarrow l$ or $l \rightarrow v$ ) capillary phase transitions occurring in the pores of PM.

It is the remarkable feature of the developed here NSG-model and its interpretation adopted by Eq.(41) that the latter corresponds congruently to the differential Clausius-Clapeyron's equation for any I-phase transition:

$$
\left(\frac{d P}{d T}\right)_{l \rightarrow v}^{I}=\frac{\delta Q}{T \delta V}=\frac{\Delta h(T)}{T \Delta v(T)} .
$$

Thus, the $T$-dependent ratio of discontinuities in the enthalpy (i.e. in the specific heat content) and the specific volume (i.e. in the inverse $(v=1 / \rho)$ mass content) reflects, as a matter of fact, the thermodynamic irreversibility of a real phase transition (!). The natural conclusion is that a spontaneous process of condensation (including its local capillary form) $v \rightarrow l$ produces quantitatively the lower cooling effect $|-\delta Q / T|$ in comparison with a concomitant process of vaporization consuming the external heat at the same temperature $(\delta Q / T)$ from Eq.(44).

The supposed hysteresis of the "latent" heat $\Delta h$ (i.e. its thermodynamically well-grounded by FT-model irreversibility between the consuming $\Delta h_{l \rightarrow v}$ and emitting
$\Delta h_{v \rightarrow l}$ heat contents) can be experimentally corroborated for any non-Gibbsian $i$-phases ( $i$ denotes $g$ (gas), v (vapor), $l$ (liquid), $s$ (solid) etc.). Their main signs and features are the finite volume $V$ and the finite time $\Delta t$ of observation in which the LE-hypothesis is applied to the heterogeneous two-phase I-phase transition's i,j-state. Both coexistent non-Gibbsian $i, j$-phases cannot (separately) be completely homogeneous in contrast to their idealized infinite Gibbsian counterparts. The typical example of a realistic heterogeneous $i, j$-coexistence for a pure fluid is, of course, the LE-states of a moist vapor (i.e. of the mechanical mixture formed by the bubbles of $v$-phase in $l$-phase or by the drops of $l$-phase in $v$-phase). This type of distribution for a mass content is a typical situation also for PMs.

FT-model [4-6] excludes (as an inaccessible result for the finite-size measurements) the reality of the strict Gibbsian equalities between the respective $i, j$-fields ( $P_{i}=P_{j}, \mu_{i}=\mu_{j}, T_{i}=T_{j}, \varphi_{i}=\varphi_{j}$ ). Thus the same constraint is also realizable for the LE-state between two actual non-Gibbsian i,j-phases. However, FT-model admits the easily verifiable by experiment with the moist air ( $m a$ ) relative stability of such mixed $i, j$-phase in the saturated density range of a 0 -phase transition: $\Delta \rho=\rho_{j}-\rho_{i}$ (see below). This relatively stable colloid (as a matter of fact) heterogeneous formation has been termed interphase (not interface (!), i.e. not the interfacial layer presumed in the standard van der Waals-Maxwell-Gibbs (WMG) theory of a unified EOS and its equilibrium phase transition [17,18]). FT-model assumes so the metastable LE-states (they exist during the finite $\Delta t$-interval) but rejects completely the unstable states of a spinodal decomposition.

This natural admission leads to the novel concept of a congruent zero-order (0) phase transition [17-21]. The standard positive characteristics for $I$-phase transitions $\chi_{T}>0$ and $C_{P}>0$ should become sometimes great but still finite ones for 0 -phase transitions. The magnitudes of these derivatives correspond to the supposed by FT-model very small disbalances between $T_{i j}$-fields $\Delta T=T_{j}-T_{i}$ at the forcedly adopted Gibbsian equality for $i j$-pressures $P_{i j}=P_{i}=P_{j}$. Vice versa, the same is true for the small disbalance: $\Delta P=P_{j}-P_{i}$ at the condition: $T_{i j}=T_{i}=T_{j}$. Thus Eq.(44) for a Gibbsian's I-phase transition should be, for example, replaced by the following equation for the 0 phase transition:

$$
\left(\frac{\Delta P}{\Delta T}\right)^{0}=\frac{\rho_{i}(\Delta h / \Delta T)_{P_{i, j}}}{\rho_{i} T_{i}(\Delta v / \Delta T)_{P_{i, j}}}=\frac{\rho_{i} C_{P}^{i j}}{T_{i} \alpha_{P}^{i j}} .
$$

Its congruence with the reference s-path of Eq.(42) is obvious. Moreover, it is straightforward task [16] to derive the similar equation for the second-order phase transition (II) in which the role of given (initial) density $\rho_{i}$ becomes pronounced:

$$
\left(\frac{\Delta P}{\Delta T}\right)^{I I}=\frac{\rho_{i} \Delta\left(C_{P}^{i j} / \rho_{i}\right)}{T_{i} \Delta\left(\alpha_{P}^{i j} / \rho_{i}\right)} .
$$

This variant of FT-model corresponds to the limiting case of an equilibrium state between two $i j$-phases occurring at 
their compatible densities and enthalpies. The respective conclusion is that the increase of pressure $(\Delta P)^{I I}>0$ alongside of the path $(d P / d T)^{I I}$ leads to the heating of medium $(\Delta T)^{I I}$ only if the both signs of discontinuities $\Delta\left(\alpha_{P}^{i j} / \rho_{i}\right)$ and $\Delta\left(C_{P}^{i j} / \rho_{i}\right)$ are the same $(+/+$ or $-/-)$. This result can be of great importance (Sect. 4) for the development of a flexible predictive NSG-model for TPMs too. In this type of PM the sharp "jumps" (discontinuities) of second thermal $\left(\chi_{T}, \alpha_{P}\right)$ and caloric $\left(C_{v}, C_{P}\right)$ derivatives in the different pores are not only possible but unavoidable.

\section{Fluctuational EOS for hydrophobic and hydro- philic TPMs}

We have assumed in Sects. 2,3 that the thermal conductivity $\lambda[\mathrm{W} / \mathrm{m} \mathrm{K}]$ and the relaxation period $\tau[\mathrm{s}]$ in Eqs.(21a,b) for two main measurable thermodynamic forces of medium ( $\nabla T$ and $\nabla P$ ) maintain their constant values while two other transport coefficient of the particle selfdiffusion $\gamma_{m}(T)$ and the electric charge conductivity $\sigma_{q}(T)$ become $T$-dependent in the proposed model of NSG. This assumption is in a complete correspondence with the Second and First Laws combined by the so-called thermodynamic EOS for single-phase i-states and twophase ij-states [18] (the subscripts $\delta \rho$ and $\delta T$ emphasize the heterogeneous nature of the 0 -phase transition):

$$
\begin{gathered}
P_{i}=T_{i}\left(\frac{\partial P_{i}}{\partial T}\right)_{\rho}+\rho_{i}^{2}\left(\frac{\partial e_{i}}{\partial \rho}\right)_{T}= \\
=T_{i}\left(\frac{\partial s_{i}}{\partial v}\right)_{T}-\left(\frac{\partial e_{i}}{\partial v}\right)_{T} \\
P_{i j}=T_{i j}\left(\frac{\partial P_{i j}}{\partial T}\right)_{\delta \rho}+\rho_{i} \rho_{j}\left(\frac{e_{i}-e_{j}}{\rho_{i}-\rho_{j}}\right)_{\delta T}= \\
=T_{i j}\left(\frac{\Delta s_{i j}}{\Delta v_{i j}}\right)_{\delta T}-\left(\frac{\Delta e_{i j}}{\Delta v_{i j}}\right)_{\delta T} .
\end{gathered}
$$

The accepted by FT-model order of priority for fields from Eq.(1) is here confirmed. Moreover, the appearance of both caloric $\Delta$-discontinuities for entropy and internal energy in Eq.(48) may become the elucidative factor for explanation of an irreversible 0-phase transition introduced for the nonGibbsian $i$,j-phases in Sect. 3. We will omit the superscript 0 and the double subscript $i j$ below to avoid the overcomplications in denotations. The fluctuation $i$-phase interpretation of thermal pressure $\left(\partial P_{i} / \partial T\right)_{\rho}=\alpha_{P}^{i} / \chi_{T}^{i}$ (see Eqs. $(41,42))$ extended here on its $i j$-phase counterpart $(d P / d T)_{\delta \rho}=\alpha_{P} / \chi_{T}$ (see Eqs. (44-46)) leads to the unambiguous conclusion. It concerns the so-called internal (energy) pressure term $\left(\partial e_{i} / \partial v\right)_{T}$ from Eq.(47) and its respective generalization $(\Delta e / \Delta v)_{\delta T}$ from Eq.(48). Both ones should be dependent on the discrete by nature variables (i.e. the numbers of particles and free electric charges). They have to be determined as the complementary parameters of a thermodynamic medium with the following exceptional role. They should compensate and relax any external irreversible changes of two main thermodynamic contributions arising due to the forces $\nabla P$ and $\nabla T$ in accordance with Eqs. $(41,42)$ and/or $(44-46)$. The result of such compensation includes the main characteristic FTparameter of relaxation $\tau$ [s]:

$$
\begin{aligned}
& \gamma_{m}(T)=\tau \cdot \rho(T, P) \quad(a) \\
& \sigma_{q}(T)=j_{q}^{2} \tau / \rho_{Q}(T, P) \quad(b)
\end{aligned}
$$

where the D'Arcy's law determines by Eq.(32) the experimental $\tau$-values[1] as the ratio of the hardly measurable permeability $K\left[\mathrm{~m}^{2}\right]$ to the purely theoretical kinematic viscosity $v\left[\mathrm{~m}^{2} / \mathrm{s}\right]$. Eq.(49) corresponds to the usual Joule's density of heat $\rho_{Q}$ which is supposedly compensated by the thermal conductivity:

$$
\sigma_{q}(T)=j_{q}^{2} \delta^{2} /(\lambda \nabla T)
$$

The derived relatively simple NSG-correlations may form the reliable basis for the creation of smart textures. Despite the widespread belief on contrary, neither $\nabla P$ nor $\nabla T$ can be directly usable as the transport governing variablesforces. Both are dependent on the changeable external conditions of TPM-exploitation. Nevertheless, it follows from our consideration that a combination of two desirable fixed flows of mass and heat $\left(j_{m}, j_{Q}\right)$ could be provided through TPM just by the appropriate choice of $\tau \nabla P$ - and $\lambda \nabla T$-magnitudes. They should be co-ordinated with the controllable variable namely of internal pressure $\left(\partial e_{i} / \partial v\right)_{T}$ or its variant $(\Delta e / \Delta v)_{\delta T}$. We intend to demonstrate below that both are determinable by the $T$ dependent cohesive $v d W$-coefficient $a_{f}(T)$ introduced in FT-EOS. In this case, the obtained explicit correlations for $\gamma_{m}(T)$ - and $\sigma_{q}(T)$-parameters in Eqs. $(32,49,50)$ become the important indicative factors to control, for example, the given optimal conditions for the comfortable wear of TPM-fabrics.

We have argued in our previous work [1] that the interrelation between the volume densities of matrix $m_{M} / V_{M}$, moist air $m_{m a} / V_{m a}$ and water content (liquid) $m_{l} / V_{l}$ is essential to specify the following factor of difference between the hydrophobic $(h b)$ and hydrophilic (hl) TPMs:

$$
\begin{aligned}
& \rho_{V}=\rho_{M}+\left(\rho_{m a}-\rho_{M}\right) V_{d a} / V+ \\
& +\left(\rho_{l}-\rho_{m a}\right) V_{l} / V
\end{aligned}
$$

where the additivity of the dry air (da) volume $\left(V_{d a}=V_{m a}+V_{l}\right)$ in the total representative volume $V=V_{d a}+V_{M}$ with the standard "dry" porosity $\varepsilon=V_{d a} / V$ have been assumed. Thus the indicative factor of above difference can be introduced by two inequalities for the a priori unknown liquid volume fraction $V_{l} / V_{d a}$ : 


$$
\begin{array}{ll}
\frac{\rho_{M}-\rho_{m a}}{\rho_{l}-\rho_{m a}}>\frac{V_{l}}{V_{d a}} \leq 1 & (\text { hb-matrix }), \\
\frac{\rho_{M}-\rho_{m a}}{\rho_{l}-\rho_{m a}}<\frac{V_{l}}{V_{d a}} \leq 1 & (\text { hl }- \text { matrix }) .
\end{array}
$$

Since both densities of TPM: $\rho_{V}$ (at the given level of relative humidity) and $\rho_{V}^{0} \approx m_{M} / V\left(\rho_{d a} \approx 0\right)$ (at the zero moisture content $\omega=0$ ) are, in principle, measurable quantities, one should, firstly, assume the following experimental estimate:

$$
\frac{V_{l}}{V_{d a}} \square \frac{\rho_{V}-\rho_{V}^{0}}{\varepsilon \cdot \rho_{l}(T)} \approx \omega[\mathrm{g} / \mathrm{kg}],
$$

where $\rho_{l}(T)$ is the saturated $T$-dependent liquid density of water. This simple assumption leads, however, to the rather interesting and useful for practice observation. The $h b$ condition in Eq.(52) can achieve the limit of its applicability with the permanent increase of moisture content $\omega(t)$. At this $t$-moment the given hb-texture becomes, formally, the modified hl-texture in which the liquid fragments may exist inside of some capillary pores. So the study of capillary condensation is necessary for both $h l$ - and $h b$-structures.

The further treatment of the wide set of experimental TPM-data in [1] has completely corroborated the result of such analysis. Indeed, the calculation of standard TPM'ssurface density $\gamma_{A}\left[\mathrm{~g} / \mathrm{m}^{2}\right]$ (where the TPM-volume is $V=A \cdot \delta$ ) has shown the existence of two quite different types of its behavior. It was revealed for the different sets of $h b$ - and $h l$-fabrics, respectively:

$$
\begin{aligned}
& \gamma_{A}^{h b}=\gamma_{0}^{h b}+\rho_{V}^{h b} \cdot \delta \quad(a) \\
& \gamma_{A}^{h l}=-\gamma_{0}^{h l}+\rho_{V}^{h l} \cdot \delta \quad(b)
\end{aligned}
$$

instead of the trivial linear $\delta$-correlation with the homogeneous TPM's-volume density $\gamma_{A}=\rho_{V} \delta$. In other words, the very small $\delta$-thickness of TPM can become in a combination with the given asymptotic parameter $\pm \gamma_{0}$ the determinative factors for its changeable properties and, first of all, permeability in accordance with the proposed in [1] alternate basis $1 / b$-model of the moist permeable media (see, in particular, Eq.(50)).

The general $f$ - and $T$-dependent FT-EOS was introduced by one of us (V.B.R.) [17] in the van der Waals (vdW)-like 3-coefficient's form:

$$
Z_{f}^{F T}-1=\frac{b_{f}(T) \rho-c_{f}(T)}{1-b_{f}(T) \rho}-\frac{a_{f}(T) \rho}{k_{B} T},
$$

where $\rho\left[\mathrm{m}^{-3}\right]$ denotes here the concentration of particles $N / V$ and $Z_{f}=P_{f}\left(\rho k_{B} T\right)$. Its further investigation and application to the wide set of problems [5,6,18-21] has corroborated its universality and high level of accuracy. The latter is achievable at the description of any $i$-phase and/or $i j$-phase states if the coexistence curve (CXC) data of I-phase transition are known from the reliable experiment $\left\{P_{s}(T), \rho_{g}(T), \rho_{l}(T)\right\}$. More accurately, such information is necessary and enough to evaluate the $T$ - dependent coefficients $\left\{a_{f}, b_{f}, c_{f}\right\}$ of $f$-phase without any adjustable parameters. In this work we propose to extend the FT-EOS' methodology (which rejects the vdW-concept of a unified (i.e. common for both coexistent $f$-phases) EOS [21]) on the description of $s$-phase (i.e. TPM's-matrix) too.

Our arguments can be discussed below for the convenience of reader in the simplified vdW-form with two constant coefficients $\left\{a_{0}, b_{0}\right\}$ :

$$
\begin{aligned}
& Z_{f}^{v d W}-1=\frac{b_{0} \rho}{1-b_{0} \rho}-\frac{a_{0}\left(b_{0} \rho\right)}{k_{B} b_{0} T}= \\
& =\frac{1-\varepsilon_{f}}{\varepsilon_{f}}-\frac{T_{B}\left(1-\varepsilon_{f}\right)}{T}
\end{aligned},
$$

where the characteristic Boyle's (B) temperature $T_{B}$ of a fluid has been introduced in terms of $B$-density $\rho_{B}$ and cohesion coefficient $a_{0}$ :

$$
T_{B}=a_{0} /\left(k_{B} b_{0}\right)=\left(a_{0} / k_{B}\right) \rho_{B} .
$$

This form defines the "dry" porosity $\varepsilon$ by means of the

ratio of free volume $\left(V-N b_{0}\right)$, where $b_{0}$ - excluded molecular volume, to the representative total volume $V$. It is the $\left(1-b_{0} \rho\right)$-parameter determined by the specific repulsive interparticle potential of $f$-phase.

But in this framework any structural aggregate properties of an excluded volume are omitted. We have used this simple observation to extend its notion on two $h b$ and $h l$-variants of $s$-phase, respectively:

$$
\begin{gathered}
Z_{s}^{h b}-1=\frac{b_{0}^{h b} \rho}{1-b_{0}^{h b} \rho}=\frac{1-\varepsilon}{\varepsilon}, \quad a_{0}^{h b}=0 \\
Z_{s}^{h l}-1=-\frac{a_{0}^{h l}}{k_{B} b_{0}^{h l}} \cdot \frac{(1-\varepsilon)}{T} .
\end{gathered}
$$

To the best of our knowledge, such postulated on the base of Eq.(56) PM-definitions are the first attempt to incorporate the physically plausible molecular-based parameters $\left(a_{0}, b_{0}\right)$ of matrix $M$ for the description of its interaction with the molecular structure of $f$-phase. An absence of cohesion contribution $a_{0}^{h b}=0$ in $h b$-textures of Eq.(59) reflects, in particular, their inability to wetting by the moist $f$-flow. The remarkable consequence of the proposed approach is a possibility to combine the given properties of $f$ - and $s$ - phases by the well-known vdWconcept of one-fluid approximation. It is most usable at the description of binary $f_{1}, f_{2}$-mixtures. We plan to represent the respective foundation of such idea in the next publications for $f$, $s$-type non-Gibbsian phases.

Some results of the discussed FT-methodology seem to be especially informative [31,32] at the application to $\mathrm{PMs}$ in the low-temperature range of water (the main component of a moisture air) located between its triple $T_{t}=273.15 \mathrm{~K}$ and normal boiling $T_{b}=373.15 \mathrm{~K}$ points. To illustrate the main 0-phase transitions approximation $\left(Z_{l} \approx 0, Z_{g} \approx 1\right)$ which becomes 
appropriate in this range, the tabular CXC-data [33] are represented in Table 1 and Fig. 1. The monotonously changeable but negligible values of $Z_{l}(T) \approx 0$ as well as the oscillating deviations of $Z_{g}(T) \approx 1$ from the ideal-gas estimate: $Z_{i g}=Z_{B}=1$ lead in accordance with Eq.(57) to the basic well-established vdW-behavior of $f$-phase [31]. Two values of free volume in $l$-phase $\pm e_{l}(T)$ are compared in Fig. 2 with that in g-phase $e_{g}(T)$ to confirm the obvious heterogeneous nature of former. This is a typical macroscopic-interphase:

$$
\varepsilon_{l}=\frac{1}{2}\left(1 \pm \sqrt{1-\frac{4 T}{T_{B}}}\right) \quad(a) \quad \varepsilon_{g}=\frac{T}{T_{B}} \quad(b) .
$$

For water the appropriate numerical estimates are [32] $T_{B}=2379[\mathrm{~K}], \quad b_{0}=0.01658 \quad\left[\mathrm{dm}^{3} / \mathrm{mol}\right]$ but the constant vdW-estimate of the cohesion coefficient $a_{0}=474\left[\mathrm{~J} \mathrm{dm} / \mathrm{mol}^{2}\right]$ seems to be rather crude for our aims. The more appropriate $T$-dependent estimate of $a_{0}(T)$ can be obtained for the low-temperature variant of FT-EOS (56) at the $I$-phase transition constraints: $b_{0}=b_{f} ; c_{0}=c_{f}=0$ and:

$$
\begin{aligned}
& a_{0}(T)=a_{g}(t)=a_{l}(T)= \\
& =-\left(e_{l}-e_{g}\right) /\left(\rho_{l}-\rho_{g}\right)
\end{aligned} .
$$

Its substitution in the universal form of thermodynamic two-phase EOS (48) gives the following system of the coupled $f$-porosities:

$$
\varepsilon_{l}=\frac{1}{Z_{g} A_{l}-1} \quad \text { (a) } \quad \varepsilon_{g}=\frac{1}{Z_{l} A_{g}+1} \quad(b) .
$$

This refined quantity is also represented in Fig. 2.

$$
\text { The cross-impact of } Z_{f}{ }^{-} \text {and }
$$

$A_{f}=\left(T / P_{s}\right)^{f}\left(d P_{s} / d T\right)^{f}$-parameters is here the very interesting feature of the proposed $\varepsilon_{f}$-estimates. It is coherent, of course, with the concept of 0-phase transition, which implies the interaction of coexistent f-phases. Such FT-correlation expressed in terms of the non-dimensional $Z_{f}$ - and $A_{f}$-factors by Eq.(63) cannot be described by the conventional WMG-theory of the I-phase transition. The latter adopts, at best, the thermodynamic reversibility of $g \rightarrow l$ and $l \rightarrow g$ VLE-transient phenomena following from the unified EOS'-concept and leading to the common $f$-coefficients of the type those from Eq.(62). Hence, the presence of different $A_{l}(T)$ - and $A_{g}(T)$-reduced slopes in the derived here Eq.(63) corresponds, namely, to the 0phase transition from Eq.(45) but not to the I-phase transition from Eq.(44) (where the equality $A_{s}(T)=A_{l}(T)=A_{g}(T)$ should be strictly fulfilled).

The fundamental distinction of the congruent CVLdiagram [5,6,18-21] from the traditional VLE-diagram requires the account for irreversibility indicated by the difference in the direct $\left(\Delta_{l, g} e(T), \Delta_{l, g} h(T)\right)$ and reverse ( $\left.\Delta e_{g, l}(T), \Delta h_{g, l}(T)\right)$ two-phase changes described by the caloric EOSs from Eq. $(11,12)$.

Table 1 - The input CXC-data [33] $\left(P_{s}, Z_{l}, Z_{g}\right)$ and FT-predicted $\left(A_{l}, A_{v}, \varepsilon_{l}, P_{h l}\right)$-parameters (see text) for the low-temperature range $\left[T_{t}, T_{b}\right]$ of water.

\begin{tabular}{|r|r|r|r|r|r|r|r|}
\hline $\boldsymbol{T}, \mathbf{K}$ & $\boldsymbol{P}_{\boldsymbol{s}}, \mathbf{k P a}$ & $\boldsymbol{Z}_{\boldsymbol{l}}$ & $\boldsymbol{Z}_{\boldsymbol{g}}$ & $\boldsymbol{A}_{\boldsymbol{l}}$ & $\boldsymbol{A}_{\boldsymbol{v}}$ & $\boldsymbol{\varepsilon}_{\boldsymbol{l}}$ & $\boldsymbol{P}_{\boldsymbol{h l}}, \mathbf{P a}$ \\
\hline 273.16 & 0.6112 & $4.845 \cdot 10^{-6}$ & 0.9993 & 15.40 & 19.84 & 0.0695 & 0.0030 \\
\hline 275.15 & 0.7054 & $5.551 \cdot 10^{-6}$ & 0.9985 & 14.58 & 19.66 & 0.0738 & 0.0039 \\
\hline 280.15 & 1.0013 & $7.739 \cdot 10^{-6}$ & 0.9989 & 14.07 & 19.22 & 0.0766 & 0.0077 \\
\hline 285.15 & 1.4016 & $1.065 \cdot 10^{-5}$ & 0.9986 & 13.80 & 18.80 & 0.0782 & 0.0149 \\
\hline 290.15 & 1.9364 & $1.447 \cdot 10^{-5}$ & 0.9984 & 13.50 & 18.39 & 0.0801 & 0.0280 \\
\hline 295.15 & 2.643 & $1.943 \cdot 10^{-5}$ & 0.9984 & 13.25 & 18.08 & 0.0818 & 0.0514 \\
\hline 300.15 & 3.564 & $2.580 \cdot 10^{-5}$ & 0.9979 & 13.05 & 17.63 & 0.0832 & 0.0919 \\
\hline 305.15 & 4.753 & $3.389 \cdot 10^{-5}$ & 0.9971 & 12.75 & 17.25 & 0.0854 & 0.1611 \\
\hline 310.15 & 6.274 & $4.409 \cdot 10^{-5}$ & 0.9972 & 12.47 & 16.90 & 0.0875 & 0.2766 \\
\hline 315.15 & 8.198 & $5.681 \cdot 10^{-5}$ & 0.9962 & 12.14 & 16.56 & 0.0901 & 0.4657 \\
\hline 320.15 & 10.614 & $7.255 \cdot 10^{-5}$ & 0.9962 & 11.96 & 16.23 & 0.0916 & 0.7700 \\
\hline 325.15 & 13.612 & $9.181 \cdot 10^{-5}$ & 0.9951 & 11.76 & 15.91 & 0.0934 & 1.2497 \\
\hline 330.15 & 17.312 & $1.153 \cdot 10^{-4}$ & 0.9941 & 11.43 & 15.60 & 0.0965 & 1.9957 \\
\hline 335.15 & 21.84 & $1.436 \cdot 10^{-4}$ & 0.9936 & 11.19 & 15.30 & 0.0988 & 3.1372 \\
\hline 340.15 & 27.33 & $1.776 \cdot 10^{-4}$ & 0.9924 & 10.95 & 15.01 & 0.1014 & 4.8537 \\
\hline 345.15 & 33.96 & $2.181 \cdot 10^{-4}$ & 0.9916 & 10.75 & 14.73 & 0.1035 & 7.4075 \\
\hline 350.15 & 41.89 & $2.660 \cdot 10^{-4}$ & 0.9904 & 10.50 & 14.46 & 0.1064 & 11.1424 \\
\hline 355.15 & 51.33 & $3.224 \cdot 10^{-4}$ & 0.9891 & 10.29 & 14.20 & 0.109 & 16.549 \\
\hline 360.15 & 62.49 & $3.883 \cdot 10^{-4}$ & 0.9876 & 10.09 & 13.94 & 0.1116 & 24.267 \\
\hline 365.15 & 75.60 & $4.649 \cdot 10^{-4}$ & 0.9861 & 9.89 & 13.69 & 0.1143 & 35.149 \\
\hline 370.15 & 90.93 & $5.537 \cdot 10^{-4}$ & 0.9844 & 9.70 & 13.46 & 0.117 & 50.351 \\
\hline 373.15 & 101.325 & $6.134 \cdot 10^{-4}$ & 0.9835 & 9.58 & 13.32 & 0.1187 & 62.156 \\
\hline 375.15 & 108.76 & $6.559 \cdot 10^{-4}$ & 0.9829 & 9.51 & 13.23 & 0.1198 & 71.333 \\
\hline & & & & &
\end{tabular}



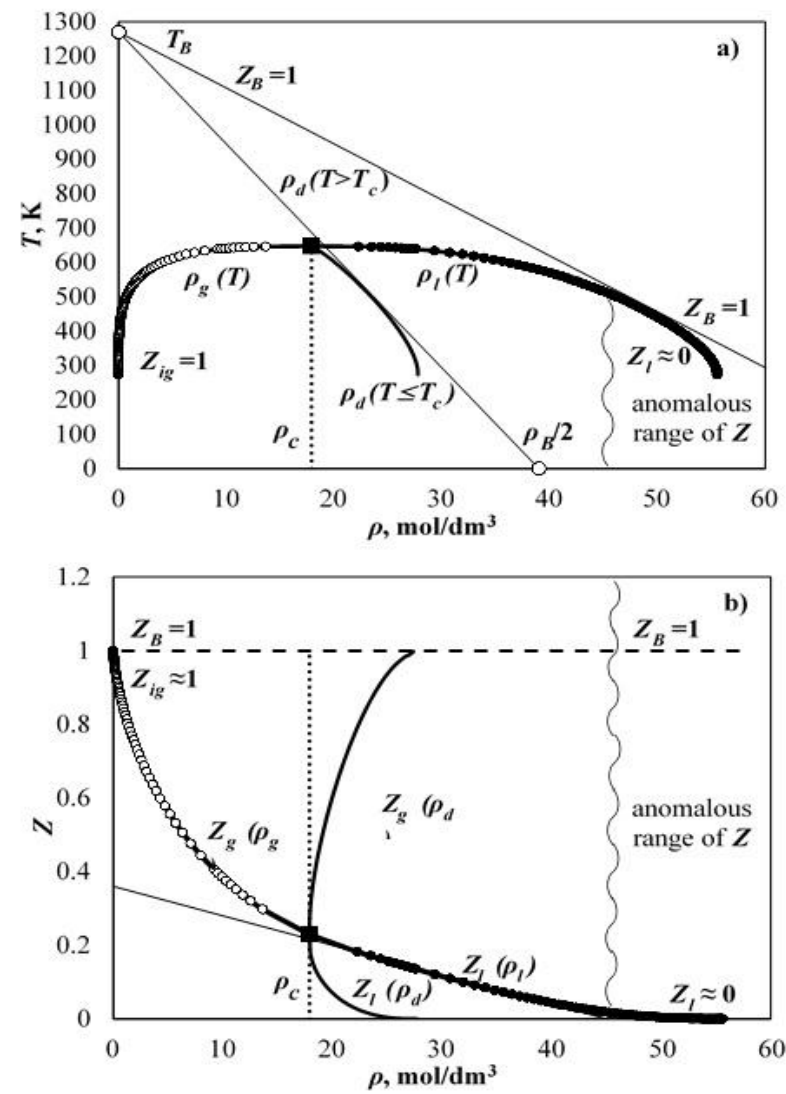

FIG.1(a) - CXC of water [33] as an example of the strongly curvilinear diameter. The formal application of Zeno-line's methodology may lead, in principle, to the serious errors at the prediction of critical parameters $\rho_{c}\left(T_{c}, P_{c}\right) .1(\mathrm{~b})-$ The elongate $\mathrm{CXC}$ and its curvilinear diameter provide the exact location of critical point although the rectilinear part of $l$ branch shown by thin line is located for $\mathrm{H}_{2} \mathrm{O}$ significantly lower than the actual critical point (black square).

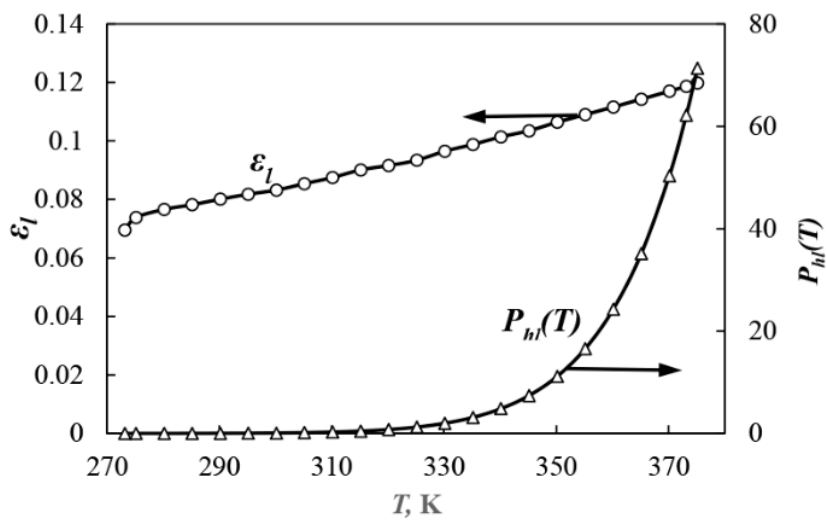

Fig. 2 - Volume fraction of liquid water $\varepsilon_{l}(T)$ in the total volume of moist air ( $\mathrm{ma}$ ) and the drop of intra-capillary pressure $P_{h l}(T)$ in the hydrophilic $(h l)$ PMs.

The important practical consequence of this requirement is the necessity to introduce the certain $\mathrm{CVL}$ corrections, especially, in the standard $(h, \omega)$-diagram [33]. It is based on the physically-plausible vdWinterpretation of $l$-phase [34]. Its main parameters are $\left(\rho_{l}, e_{l}\right)$ while $s_{l} \rightarrow 0$. This concept leads to the choice of zero-point for entropy of $l$-phase $s_{l}\left(T_{t}=273.16 K\right)=0$ at the triple point of $\mathrm{H}_{2} \mathrm{O}$ and to the ideal-gas'-estimate of its enormous value in $g$-phase: $h_{i g}\left(T_{t}\right)=T_{t} s_{i g}\left(T_{t}\right)+h_{l}\left(T_{t}\right)$, where $h_{i g} \square h_{l}\left(T_{t}\right) \approx 0$. Just the rather arbitrary and singular estimate of $h_{i g}\left(T_{t}\right) / s_{l}\left(T_{t}\right)$-ratio at the triple point corresponds to the slope of reference isoenthalpy $h_{m a}=q$ in the $(T, \omega)$ plane at the construction of standard $(h, \omega)$-diagram.

We propose to take into account that the experimental value of the consumed vaporization heat:

$$
\begin{aligned}
& \Delta_{l, g} h=h_{g}\left(T_{b}, P_{s}=0.101 M P a\right)- \\
& -h_{l}\left(T_{b}, P_{s}=0.101 M P a\right) \approx 2.26 \mathrm{~kJ} / \mathrm{kg}
\end{aligned}
$$
measurable parameter at the adjustable determination of the above initial value [33] for water: $h_{i g}\left(T_{t}\right)=2.5 \cdot 10^{3}$ $[\mathrm{kJ} / \mathrm{kg}]$. FT-model states that the respective latent heat $\Delta_{g, l} h$ of the reverse condensation process $(g \rightarrow l)$ is always significantly less for the real 0 -phase transitions at which, additionally, $P_{l}(T)<P_{v}(T)$. Hence, the introduction of such correction at the standard construction of $(h, \omega)$-diagram may be, in principle, the essential factor of adequacy for the description of any condensation phenomena including capillary one.

In this work we have developed the rather simple and useful estimate of the intra-porous pressure $P_{h l} \leq P_{S}(T)$ within $h l$-systems. It is based on the previous results and admits the following combination $[18,36]$ of the wellknown correlations introduced long ago [37] by Laplace and Kelvin:

$$
P_{h l} / P_{s}(T)=\exp \left\{Z_{l}(T)\left[1-P_{s}(T) / P_{h l}(T)\right]\right\} .
$$

Its evident advantage is an absence of any hardly measurable for PMs and TPMs parameters of surface tension and fractal geometry of a dividing intra-capillary surface. The capillary condensation is formally impossible in $h b$-textures for which $P_{h b}>P_{s}(T)$.

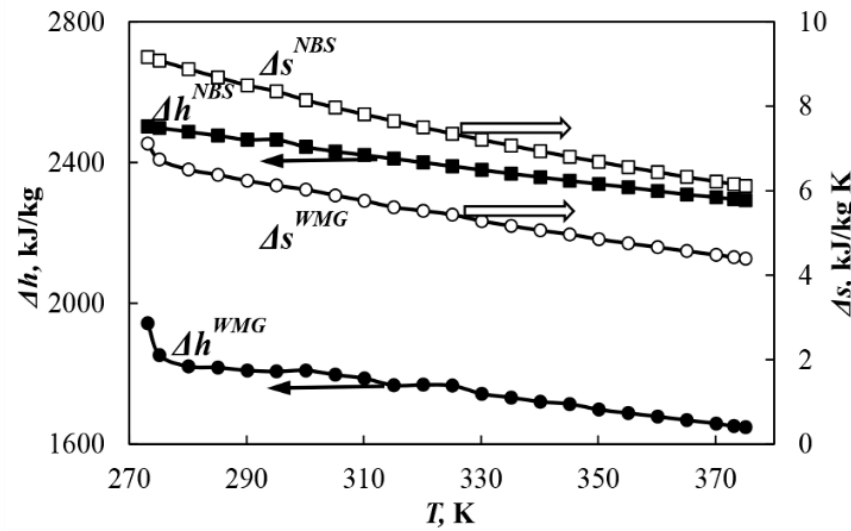

Fig. 3 - FT-calculated caloric functions of discontinuities in the enthalpy and entropy compared with the classical WMG-estimates in PMs.

To solve this transcendent equation we have used its differential form, which immediately leads to the 
remarkably simple estimate (shown also in Fig. 2) for the sought-for intra-porous pressure:

$$
P_{h l}=P_{s}(T) \cdot Z_{l}(T) \text {. }
$$

Hence, the independent $P_{h l}(T)$-estimate may be the first step to use the discussed in this work hierarchy of fields and the developed NSG-model in accordance with the derived in Sect. 1-4 resulting equations of a combined mass-heatcharge transport.

\section{Conclusion}

The proposed NSG-model opens the new wide field of the relevant investigations in the non-equilibrium thermodynamics due to the formal absence of an $x$ integration and of its usual complexities. We consider that only $t$-dependence of indicative parameter are essential at the description of transport processes through TPMs.

\section{References}

1. O.V. Rogankov Jr., M.V. Shvets and V.B. Rogankov, Alternate basic $l / b$-model of effective porosity created for hydrophilic $(l)$ and/or hydrophobic (b) moist textile materials, Fibres and Textiles in Eastern Europe 2016; 24, 3(117): 51-57. DOI http://dx.doi.org/10.5604/12303666.1196612.

2. I. Dyarmati, Non-equilibrium thermodynamics. Fluid theory and variational principles, Mir, M., 1974.

3. P. Glansdorf and I. Prigogine, Thermodynamic theory of structure, stability and fluctuations, Mir, M., 1973.

4. V.B. Rogankov and V.K. Fedyanin, Fluctuational theory of media with strong space-time inhomogeneity, Theor. and Math. Phys, v.97, 53-67 (1993).

5. V.B.Rogankov and V.I.Levchenko, Global asymmetry of fluids and local singularity in the diameter of the coexistence curve, Phys.Rev.E 87, 052141 (2013).

6. V.B. Rogankov, Fluctuational-thermodynamic interprettation of small angle X-ray scattering experiments in supercritical fluids, Fluid Phase Equilibria 383, 115-125 (2014).

7. D.A. de Vries, The theory of heat and moisture transfer in porous media revisited, Int. J. Heat and Mass Transfer, v.7, 1343-1350 (1987).

8. K.Vafai, Handbook of porous media, Tayler and Francis, NY, USA, 2005.

9. S.Larbi, Heat and mass transfer with condensation in capillary porous bodies, Hindawi Publ.Corpor., The Scientific World Journ., v.2014, Art ID 194617, 8.

10. ISO 9346: 2007 "Hydrothermal performance of buildings and building materials - Physical quantities for mass transfer.-Vocabulary.

11. M.Janz-Lund, Method of measuring the moisture diffusivity at high moisture levels, Division of building materials - Report TVBM-3076-1997. 76 p.

12.H.M.Kunzel and K.Kiessi, Calculation of heat and moisture transfer in exposed building components, Int.J of Heat and Mass Transfer, 1997, v.40, № 1, pp.159-167.

13. M.I.Nizovtsev, S.V.Stankus, A.N.Sterkyagov, V.I.Terekhov, R.A.Khairullin, Determination moisture diffusivity in porous building materials using gammamethod, Int.J of Heat and Mass Transfer, 2008, v.51, Issues 17-18, pp.4161-4167.
14. N.V.Pavlukevich, Introduction to theory of heat- and mass transfer in porous media - Minsk, Inst. of Heat and Mass Exchange - NANB, 2002, p.140.

15. M.I.Nizovtzev, A.N.Sterliagov, V.I.Terehov, Verification of model for calculation of coupled thermal and moisture transfer at the humidity of gas-concrete - Izv. Vyzov. Building - 2008, № 1, p. 104, 2008.

16. U.B.Rumer and M.Sh.Ryvkin, Thermodynamics, Statistical Physics and Kinetics, Nauka, M., 1972.

17. V.B.Rogankov and L.Z.Boshkov, Gibbs solution of the van der Waals-Maxwell problem and universality of the liquid-gas coexistence curve, Phys.Chem.Chem.Phys. 4, 873-878 (2002).

18. V.B.Rogankov, Asymmetry of heterophase fluctuations in nucleation theory, in Nucleation Theory and Applications (edited by J.W.P.Schmelzer, G.Röpke and V.B.Priezjev) Chapt. 22, Dubna, JINR, 2011.

19. V.B.Rogankov and V.I.Levchenko, Towards the equation of state for neutral $\left(\mathrm{C}_{2} \mathrm{H}_{4}\right)$, polar $\left(\mathrm{H}_{2} \mathrm{O}\right)$, and ionic ([bmim $\left.]\left[\mathrm{Bf}_{4}\right], \quad[\mathrm{bmim}]\left[\mathrm{Pf}_{6}\right], \quad[\mathrm{pmmim}]\left[\mathrm{Tf}_{2} \mathrm{~N}\right]\right)$ liquids, Journal of Thermodynamics, Volume 2014, Article ID 496835, 15 pages, http://dx.doi.org/10.1155/2014/496835

20. V.B.Rogankov, Scaling Model of Low-Temperature Transport Properties for Molecular and Ionic Liquids, Journal of Termodynamics, Volume 2015, Article ID 208486, 11 pages, http://dx.doi.org/10.1155/2015/208486

21. O.V.Rogankov Jr. and V.B.Rogankov, Can the Boyle and critical parameters be unambiguously correlated for polar and associating fluids, liquid metals, ionic liquids? Fluid Phase Equilibria 2017, 434, 200-210 http://dx.doi.org/10.1016/j.fluid.2016.11.034

22. C.L.Tucker III and R.B.Dessenberger, Governing equation for flow and heat transfer in stationary fiber beds, in S.G.Advani, ed. Flow and Rheology in Polymer Composites Manufacturing, Chapt. 8, N.-Y., Elsevier Sci., 1994, pp. 257-323.

23. R.G.Carbonell and S.Whitaker, Dispersion in pulsed systems II. Theoretical developments for passive dispersion in porous media, Chem. Eng. Sci. 38, 1795, 1983.

24. S.G.Advani and K.-T.Hsiao, Transport phenomena in liquid composites molding processes and their roles in process control and optimization, in K.Vafai ed. Handbook of porous media (see [8]) pp. 573-606.

25. A.Amiri and K.Vafai, Transient analysis of incompressible flow through a packed bed, Int. J. Heat and Mass Transfer 41, 4259, 1998.

26. F.Zanotti and R.G.Carbonell, Development of transport equation for multiphase system I-III, Chem. Eng. Sci. 39, 263, 1984.

27. M.Quintard and S Whitaker, One and two equation models for transient diffusion processes in two phase systems, Advan. Heat. Transfer 23, 269, 1993.

28.S Whitaker, The method of volume averaging, Dordrecht, Kluwer, 1999.

29. A.V.Kuznetsov, Thermal nonequilibrium forced convection in porous media, in D.B.Ingham and I.Pop, eds. Transport Phenomena in Porous Media, Amsterdam; Elsevier, 1998, pp.103-129.

30. A.S.Iberall, Permeability of glass wool and other highly porous media, Rep. Bur. Aeronautics Navy Depart., Washington, DC, USA, 398-406.

31. V.B.Rogankov, V.I. Levchenko and Y.K. Kornienko, Fluctuational equation of state and hypothetical phase 
diagram of super-heated water and two imidazolium-based ionic liquids, J. of Mol. Phys., 02919 (2009), p.1-6.

32. V.B.Rogankov, Disorder parameter, asymmetry and quasibinodal of water at negative pressures, In "Metastable states of simple and complex compounds", edited by S.Rzoscka and V.A.Mazur; NATO Sci Ser S. Publ.2009, pp.21-27.

33. A.Saul and W.Wagner, J. International Equations for the saturated properties of ordinary water substance, Phys. Chem. Ref. Data: 16, 893, 1987.

34. W.G.Hoover, G.Stell, E.Goldmark and G.D.Degani, Generalized van der Waals equation of state, Chem.Phys. 63, 5434-5438 (1975).
35. J.O.Hirschfelder, C.F.Curtiss and B.B.Bird, Molecular Theory of Gases and Liquids, J.Wiley and Sons, N.Y., 1954.

36. V.B.Rogankov, O.G.Byutner, T.A.Bedrova and T.V.Vasiltsova, Local phase diagram of binary mixtures in the near-critical region of solvent, J.Molec.Liq., 127, 53-59 (2006).

Received 05 July 2017 Approved 08 September 2017 Available in Internet 30 October 2017 